www.nature.com/pj

\title{
Selective chiral handedness induced in spin-coated polyaramide films
}

\author{
Kunihiko Okano and Takashi Yamashita \\ Polymer Journal (2011) 43, 941-943; doi:10.1038/pj.2011.89; published online 21 September 2011
}

Keywords: CD spectra; optical activity; polyaramide; supramolecular chirality

Supramolecular structures are formed by noncovalent interactions such as electrostatic interactions, hydrogen bonding, van der Waals contacts and $\pi-\pi$ interactions. Therefore, controlling these noncovalent bonds is an efficient approach to the construction of precise chiral structures. Usually, chiral supramolecular assemblies have been built from chiral molecules or building blocks. ${ }^{1,2}$ In specific cases, chiral assemblies can also be obtained from achiral molecules through noncovalent interactions. ${ }^{3,4}$ In recent studies, vortices showing a macroscopic chirality have also been regarded as sources of chirality. ${ }^{5}$ In fact, electrostatic $J$-aggregation of a 4 -sulfonatophenylporphyrin in aqueous media has demonstrated that the sense of optical activity can be selected using the direction of rotary stirring. ${ }^{6-9}$ The optical activity of a helical ribbon reflected the direction of the chirality induced in a stirred solution. ${ }^{9}$ Furthermore, a hydrogen-bonded dendritic porphyrin wire gives a selective and reversible optically active solution, suggesting that rotational stirring is a potential tool for chiral induction. ${ }^{10,11}$ The dendritic porphyrin derivatives form optically active films whose enantiometric form is determined by the spinning direction. ${ }^{11}$ The noncovalent interaction of these porphyrin systems and hydrodynamic effects has an important role in selecting chiral handedness.

We investigated the possibility of a selective induction of optical activity using an artificial polymer system (Figure 1a), which exhibits a lyotropic liquid crystalline (LLC) phase in water. As mentioned above, achiral porphyrin derivatives have successfully formed optically active assemblies from fluid vortexes and spin-coated solutions by controlling their $J$-aggregation through $\pi$-stacking interactions. ${ }^{12}$ Herein, we found that the polyaramide films fabricated by spin-coating show optical activity and that their handedness is selected by the spinning direction. Spinning the films changes the direction of the hydrogen bonds that form between the polymers. The simple chemical structure of the polyaramide obtained through a short synthetic procedure makes it useful as a chiral template material.

The sulfonated polyaramide (PPSA) used in this study was synthesized by polycondensation between 2-sulfoterephthalic acid and 1, 4-phenylenediamine. ${ }^{13}$ Despite a rigid structure composed of main- chain aromatic and amide groups, PPSA was dissolved in water because of the hydrophilic inverted sulfonic acid. The solutions became viscous over $2.0 \mathrm{wt} \%$ of PPSA and changed into gels when the concentration of solution reached $3.0 \mathrm{wt} \%$ (Figure $1 \mathrm{~b}$ ). When viewed with polarizing optical microscopy, the sample solutions showed typical LC (liquid crystal) textures (Figure 1c) for concentrations exceeding the critical value of $0.6 \mathrm{wt} \%$. The gelled samples also showed an LC phase, suggesting that hydrogen bonding participates in the construction of a highly ordered LC phase.

We used circular dichromism (CD) spectroscopy to probe the degree of optical activity of the spin-coated films and their handedness (Figure 2). Film specimens containing $0.5 \mathrm{wt} \%$ to $3.0 \mathrm{wt} \%$ of PPSA were prepared. Films prepared from aqueous lyotropic LC solutions were optically active, whereas the ones prepared from isotropic solutions were silent in the CD spectroscopy. For example, a resultant sample film formed by spin-coating a $3.0 \mathrm{wt} \%$ PPSA solution in a clockwise direction at 3000 r.p.m. shows intense CD bands at $235 \mathrm{~nm}$ ( $-814 \mathrm{mdeg})$ and $356 \mathrm{~nm}$ ( $1440 \mathrm{mdeg}$; Figure 2a; blue curve). In contrast, spin-coating the same solution in a counterclockwise direction generates the mirror image of CD spectrum (Figure 2a; red curve). To further support these results, the statistical distribution of the data from 10 trials for each sample concentration and spinning direction (insets in Figure 2) was calculated. These statistical data show a complete dominance of the emerging chirality sense. In addition, the distributions of CD signal maxima and their wavelength suggested differences in the molecular packing density and direction (Supplementary Figure S1). The data also show that the absolute ellipticity values increase with increasing concentration of the PPSA solution used for the film preparation. On the other hand, the sample films prepared from the isotropic samples, which correspond to 0.5 $w t \%$ PPSA solutions, did not show any optical activity on spin-coating (Figure 2d). The optically active films showed the same CD spectra after several months of storage at an ambient temperature. Moreover, their optical activity was preserved on heating at $200^{\circ} \mathrm{C}$ for $5 \mathrm{~h}$.

Next, we investigated the relationship between the films' optical activity and their molecular alignment using ultraviolet-visible 
<smiles>CC(C)(C)Nc1ccc(NC(=O)c2ccc(C(=O)C(C)(C)C)c(S(=O)(=O)O)c2)cc1</smiles>

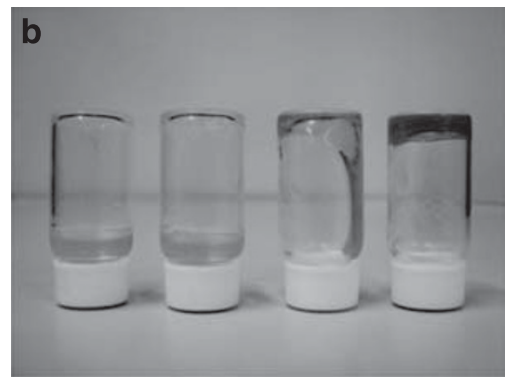

C
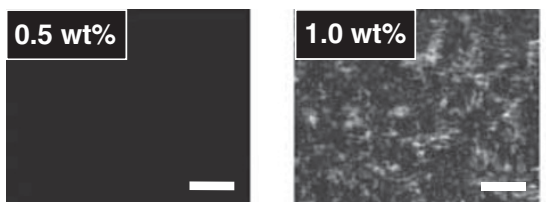
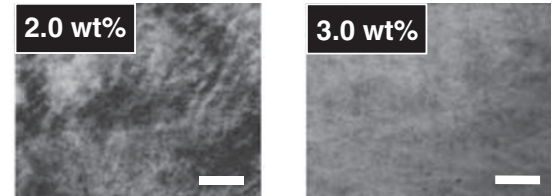

Figure 1 (a) Schematic molecular structure. (b) Optical image, and (c) polarizing optical micrographs of 0.5, 1.0, 2.0 and 3.0 wt\% of aqueous sulfonated polyaramide (PPSA) solutions (left to right). Scale bar: $50 \mu \mathrm{m}$. A full color version of this figure is available at Polymer Journal online.
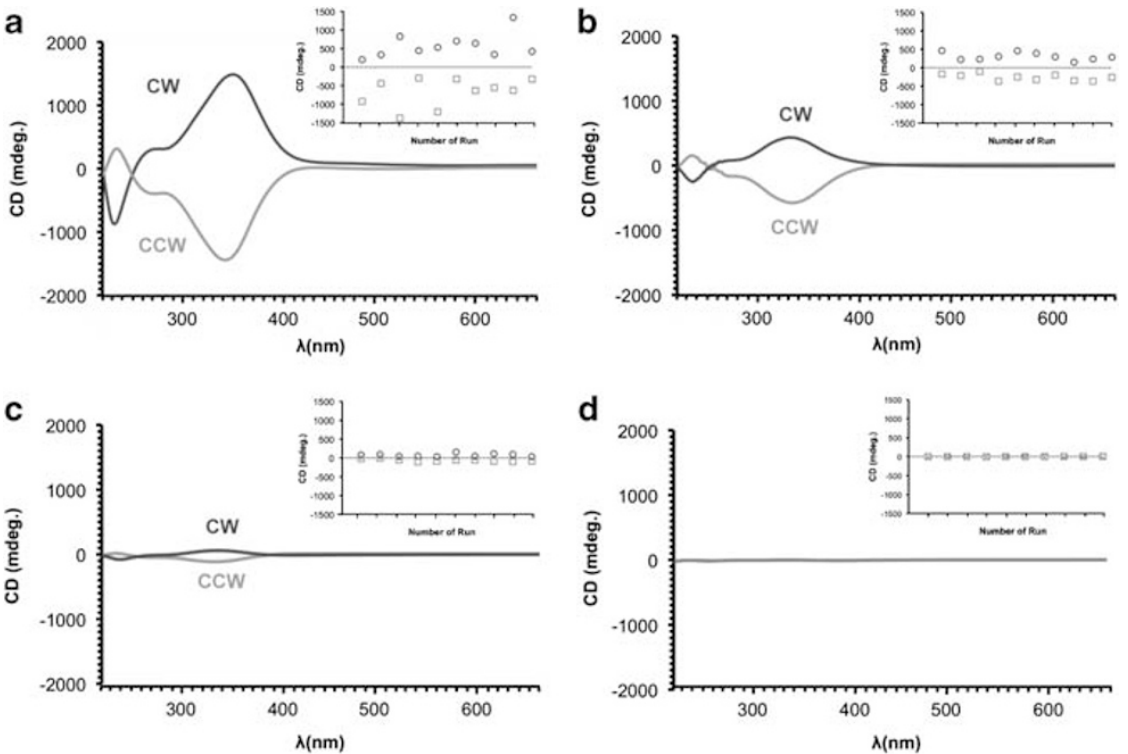

Figure 2 The films prepared by spin-coating in clockwise (CW) and counterclockwise (CCW) directions at 3000 r.p.m. for 60 s on a quart substrate. The films were obtained from different aqueous sulfonated polyaramide (PPSA) solutions: (a) 3.0 wt\%; (b) 2.0 wt\%; (c) 1.0 wt $\%$; and (d) 0.5 wt $\%$ (insets). The statistical distributions at $350 \mathrm{~nm}$ were calculated from the data of 10 trials for each PPSA concentration and spinning direction (blue circles: clockwise spinning; red squares: counterclockwise spinning). CD, circular dichromism. A full color version of this figure is available at Polymer Journal online.

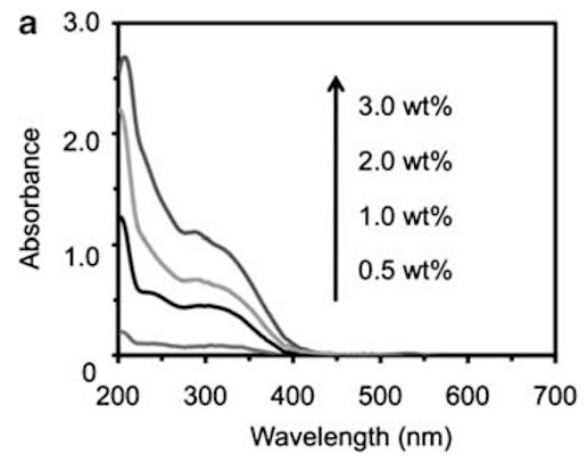

b
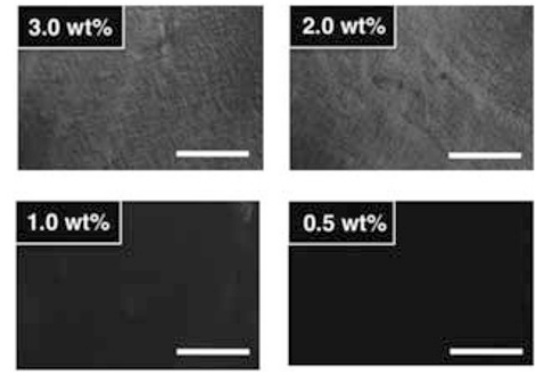

Figure 3 (a) Absorption spectra of the films spin-coated from aqueous sulfonated polyaramide (PPSA) solutions (3.0 wt $\%$, blue line; 2.0 wt $\%$, red line; 1.0 wt $\%$, black line; and 0.5 wt $\%$, green line). Film thicknesses: 3.0 wt $\%, 400 \mathrm{~nm} ; 2.0 \mathrm{wt} \%, 200 \mathrm{~nm} ; 1.0 \mathrm{wt} \%, 150 \mathrm{~nm}$; and $0.5 \mathrm{wt} \%, 30 \mathrm{~nm}$. (b) Polarizing optical micrographs of the films prepared from $3.0 \mathrm{wt} \%, 2.0 \mathrm{wt} \%, 1.0 \mathrm{wt} \%$ and $0.5 \mathrm{wt} \%$ of PPSA solutions. Scale bar: $50 \mu \mathrm{m}$. A full color version of this figure is available at Polymer Journal online. 
spectroscopy and polarizing optical microscopy (Figure 3). The absorption spectra show a broad band between 290 and $390 \mathrm{~nm}$, which coincides with the CD signal region (Figure 3a). This band is characteristic of the $\pi$-stacking of benzene, a hydrogen-bonded structure. Polarizing optical microscopy shows intense birefringence in the sample films spin-coated from LLC phases, which exhibited optical activity (2.0 wt $\%$ and $3.0 \mathrm{wt} \%$ PPSA; Figure $3 \mathrm{~b}$ ). This result suggests that the LC molecular order in the film is preserved after spinning. On the other hand, the films spin-coated from $1.0 \mathrm{wt} \%$ PPSA solutions show weak CD signals and little birefringence, which is consistent with a loss in LC order due to rotational force. The films spin-coated from isotropic sample solutions (0.5 wt\% PPSA) showed no birefringence. The absorption spectra and polarizing optical microscopy suggest that the hydrogen-bonded LC alignments preserve a large rotational shear force and generate optical activity depending on PPSA concentrations. A rotational rate dependency was observed (Supplementary Figure S2), which means a shearing force could reflects the optical activity. Moreover, position-dependent CD intensity distributions occurred; that is, the center of spinning gave rise to intense $\mathrm{CD}$ bands (Supplementary Figure S3).

The CD spectra give rise to signs that originated not only from chirality but also from linear artifacts. ${ }^{14,15}$ In our system, the artifactual elements would hamper the analysis of CD spectra, because the sample films were prepared from a lyotropic LC exhibiting birefringence. In a recent study, a measurement taken with polarizing optical microscopy demonstrated mechanically induced chirality in an LLC system, ${ }^{16}$ which implies that the films used in this study possessed chirality. In fact, the handedness of the $\mathrm{CD}$ signals can be selected by the spinning direction. Therefore, we postulate that the films memorize some chirality that originated from a mechanical rotation even though the linear artifact is also comprised in it.

With the above observations in mind, we proposed a potential mechanism for the formation of optically active films through spincoating. As mentioned previously, hydrogen bonding, which determines the three-dimensional order of the polymer chains, may have a key role in inducing chirality according to the spinning direction. The formation of supramolecular assemblies requires multipoint noncovalent interactions. In our system, each monomer unit has amide moieties, which form hydrogen bonds, indicating that polymer chains have multipoint hydrogen bonding sites. Because of these multipoint sites of hydrogen bonding, the polymer backbones form helically twisted structures by spinning. The polymer backbones then memorize a supramolecular chiral structure, depending on the direction of rotation, and even contain some artifacts from birefringence. ${ }^{14}$

This study describes the first synthesis of optically active films obtained from the aqueous LLCs of synthetic achiral polymers. In this system, hydrogen bonding between a polymer backbone and amide groups is important in inducing chirality by mechanical rotation. Moreover, the polyaramide LLC may be used as a template for asymmetric synthesis. The LLC sample is mostly composed of water (9798\%), and the polyaramide is obtained through short and easy synthetic procedures. Therefore, this LLC system, which exhibits the potential to control chiral handedness by mechanical rotation, is useful for versatile applications due to its processability and environmental sustainability.

\section{CONFLICT OF INTEREST}

The authors declare no conflict of interest.

\section{ACKNOWLEDGEMENTS}

This work was supported by the second stage of CLUSTER from the Ministry of Education, Culture, Sports, Science and Technology, Japan.

1 Rowan, A. E. \& Nolte, R. J. M. Helical molecular programming. Angew. Chem. Int. Ed. 37, 63-68 (1998).

2 Hirschberg, J.H.K.K., Brunsveld, L., Ramzi, A., Vekemans, J. A., Sijbesma, R. P. \& Meijer, E. W. Helical self-assembled polymers from cooperative stacking of hydrogenbondedpairs. Nature 407, 167-170 (2000).

3 Ohno, O., Kaizu, Y. \& Kobayashi, H. J-aggregate formation of a water-soluble porphyrin in acidic aqueous media. J. Chem. Phys. 99, 4139 (1999).

4 De Rossi, U., Dähne, S., Meskers, S. C. J. \& Dekkers, H. P. J. M. Spontaneous formation of chirality in J-aggregates showing davydov splitting. Angew. Chem . Int. Ed. 35, 760-763 (1996).

5 Kostur, M., Schindler, M., Talkner, P. \& Haggi, P. Chiral separation in microfluids. Phys. Rev. Lett. 96, 014502 (2006).

6 Ribó, J. M., Crusats, J., Sagués, F., Claret, J. \& Rubires, R. Chiral sign induction by vortices during the formation of mesophases in stirred solutions. Science 292, 2063-2066 (2001).

7 Escudero, C., Crusats, J., Díez-Pérez, I., Hachemi, Z. E. \& Ribó, J. M. Folding and hydrodynamic forces in J-aggregates of 5-phenyl-10,15,20-tris(4-sulfophenyl)porphyrin spontaneous formation of chirality in J-aggregates showing davydov splitting. Angew. Chem. Int. Ed. 35, 8032-8035 (2006).

8 El-Hachemi, Z., Arteaga, O., Canillas, A., Crusats, J., Escudero, C., Kuroda, R., Harada, T., Rosa, M. \& Ribo, J. M. On the mechano-chiral effect of vortical flows on the dichroic spectra of 5-phenyl-10,15,20-tris(4-sulfonatophenyl)prophyrin J-aggregates. Chem. Euro. J. 14, 6438-6443 (2008).

9 Arteaga, O., Canillas, A., Crusats, J., Escudero El-Hachemi, Z., Llorens, J. \& Ribo, J. M. Emergence of supramolecular chirality by flows. Chemphyschem 11, 3511-3516 (2010).

10 Tsuda, A., Alam, M. A., Harada, T., Yamaguchi, T., Ishii, N. \& Aida, T. Spectroscopic visualization of vortex flows using dye-containing nanofibers. Angew. Chem. Int. Ed. 46, 8198-8202 (2007).

11 Wolffs, M., George, S. J., Tomovic, Z., Meskers, S. C., Schenning, A. P. H. J. \& Meijer, E. W. Macroscopic origin of circular dichroism effects by alignment of self-assembled fibers in solution. Angew. Chemie. Int. Ed. 46, 8203-8205.

12 Yamaguchi, T., Kimura, T., Matsuda, H. \& Aida, T. Macroscopic spinning chirality memorized in spin-coated films of spatially designed dendritic zinc porphyrin J-aggregates. Angew. Chem. Int. Ed. 43, 6350-6355 (2004).

13 Davis, V. A., Viale, S., Li, N., Schotman, A. H. M., Best, A. S., Picken, S. J. Synthesis and formation of a supramolecular nematic liquid crystal in poly(p-phenylene-sulfoterephthalamide) $\mathrm{H}_{2} \mathrm{O}$. Macromolecules 38, 3647-3652 (2005).

14 Kuball, H.- G. \& Altschuh, J. Optical activity of oriented molecules comparision of the optical rotatory dispersion and the circular dicchroism through the kramers-kronig transform. Chem. Phys. Lett. 87, 599-603 (1982).

15 Povel, H., Aebersold, H. \& Stenflo, J. O. Charge-coupled device image sensor as a demodulator in a 2-D polarimeter with a piezoelastic modulator. Appl. Opt. 29, 1186-1190 (1990).

16 Tortora, L. \& Lavrentovich, O. D. Chiral symmetry breaking by spatial confinement in tactoidal droplets of lyotropic chromonic liquid crystals. PNAS 108, 5163-5168 (2011).

17 Okano, K., Arteaga, O., Ribo, J. M. \& Yamashita, T. Emergence of chiral environments by flows; the case of an ionic oligomer and congo red dye. Chem. Eur. J. 17, 9288-9292 (2011). 